\title{
Identification des Systèmes de Connaissances Technologiques Défense au sein des industries A\&D.
}

\section{Identification of Technological Knowledge Systems in the A\&D Industries.}

\author{
François-Xavier Meunier ${ }^{1}$ \\ ${ }^{1}$ Laboratoire Unité d'économie appliquée (UEA), ENSTA ParisTech, francois-xavier.meunier@ensta-paristech.fr
}

\begin{abstract}
RÉSUMÉ. L'objectif de cet article est de proposer une méthode pour repérer empiriquement les systèmes de connaissances qui structurent le " paysage technologique » au sein des industries de défense. Pour ce faire, cet article propose de mettre en évidence des Systèmes de Connaissances Technologiques (SCT). Isoler les SCT permet de mettre en évidence non seulement les briques de connaissances (component knowledge) mais aussi les architectures de connaissances (architectural knowledge) qui, pour chaque système, structurent la dynamique d'innovation dans le domaine de la défense.

ABSTRACT. The purpose of this article is to offer a method for empirically identifying the knowledge systems that structure the "technological landscape" within the defense industries. In order to do this, this article proposes shedding light on Technological Knowledge Systems. Isolating these systems makes it possible to shed light on not only component knowledge but also architectural knowledge, which, for each system, structures innovation dynamics in the defense sector.

MOTS-CLÉS. Système, Innovation Technologique, Brevet, Connaissance.

KEYWORDS. System, Technological Innovation, Patent, Knowledge.
\end{abstract}

\section{Introduction}

Depuis les années 1980 et l'émergence des débats sur les retombées technologiques entre la sphère civile et la sphère de défense [ALI 92] puis le développement du concept de dualité (p.ex. : [GUM 88]), la séparation, plus ou moins légitime, entre technologie militaire et technologie civile est devenue plus ténue. Les dynamiques d'innovation au sein de la Base Industrielle et Technologique de Défense (BITD) sont aujourd'hui largement influencées par celles qui s'exercent au sein des industries civiles [MEU 17]. Identifier quelles sont les dynamiques d'innovation propres au domaine de la défense au sein de la BITD est devenu plus complexe. Or, en dépit de cette proximité, identifier ces dynamiques reste un enjeu pour les pouvoirs publics et les industriels. Ces derniers doivent non seulement identifier les technologies nécessaires au développement des systèmes de défense d'aujourd'hui, mais aussi veiller au maintien des compétences et des connaissances essentielles aux systèmes de demain. Sans compter que la bonne connaissance du périmètre technologique de la défense ainsi que ses caractéristiques constituent une information nécessaire à l'intégration de l'innovation de défense à un périmètre technologique de plus en plus dual et mondialisé.

L'objectif de cet article est de proposer une méthode pour repérer empiriquement les systèmes de connaissances qui structurent le paysage technologique au sein des industries de défense. Pour ce faire, cet article propose de mettre en évidence des Systèmes de Connaissances Technologiques (SCT). Isoler les SCT permet de mettre en évidence non seulement les briques de connaissances (component knowledge) mais aussi les architectures de connaissances (architectural knowledge) qui, pour chaque système, structurent la dynamique d'innovation dans le domaine de la défense.

Dans un premier temps cet article reviendra sur l'intérêt d'identifier les systèmes de connaissances au coeur de l'innovation de défense. Puis la méthode d'identification sera détaillée avant de présenter les résultats obtenus. 


\section{Définition des SCT et innovation de défense}

Cet article propose une méthode originale permettant d'analyser la base de connaissances des firmes. Elle s'articule autour du concept, défini dans cette section, de «Systèmes de Connaissances Technologiques » (SCT) et tire profit des informations contenues dans les dépôts de brevets. L'objectif de ce cette méthode est de s'émanciper de l'étude de cas, communément employée afin d'étudier empiriquement la production technologique dans le secteur de la défense. Pour cela, la méthode repose sur l'étude des connaissances qui constituent un élément de base de l'innovation technologique [CAR 91].

La technologie est abordée au travers d'une perspective systémique, ceci afin de mieux comprendre les multiples modalités d'interaction technologique entre la sphère civile et la sphère militaire. En effet c'est en considérant le système technologique dans sa globalité que l'on est à même de mesurer la complexité des connaissances qui le constituent

Le système technologique a été défini par Carlsson et Stankiewicz: «A technological system may be defined as a network of agents interacting in a specific economic/industrial area under a particular institutional infrastructure or set of infrastructures and involved in the generation, diffusion, and utilization of technology. Technological systems are defined in terms of knowledge/competence flows rather than flows of ordinary goods and services. » [CAR 91]. Quatre principaux éléments composent les systèmes technologiques :

- Un noyau dur de connaissances techniques et scientifiques.

- Une constellation de systèmes techniques.

- Un environnement de marché.

- Une interface institutionnelle.

Cette représentation du système technologique semble tout à fait appropriée pour mieux comprendre l'intégration de la défense dans un paysage technologique plus large. En effet, elle montre qu'une même base de connaissances peut donner lieu au développement de différents produits qui peuvent donc dépasser la sphère de défense ou encore qu'un même produit peut nécessiter une combinaison de connaissances disponibles au-delà de la sphère au sein de laquelle il a émergé. En effet, elle montre que différents produits peuvent adresser différents consommateurs alors qu'ils reposent sur des technologies construites à partir d'une base de connaissances techniques et scientifiques semblables (représentée par le grand rectangle gris). Ainsi, dans le cas présent, soit une (ou plusieurs) technologie(s) peut donner lieu à plusieurs produits distincts qui sont chacun destinés au domaine militaire ou au domaine civil ; soit, un produit reposant sur une (ou plusieurs) technologie(s) permet de développer un produit destiné à la fois aux militaires et aux civils. Dans les deux cas c'est un ensemble de connaissances qui, combinées d'une certaine manière, permettent de faire le pont entre les domaines civil et militaire.

Un concept connexe mais néanmoins distinct est celui de système technique [GIL 78]. Lui non plus ne s'attache pas seulement aux caractéristiques techniques ou secteurs auxquels un système est rattaché mais cherche plus particulièrement à comprendre comment les éléments d'un système (acteurs, organisations, connaissances, applications, usages) s'organisent. Dans son analyse, il met en avant le rôle des technologies dites génériques, autrement appelées General Purpose Technologies, [BRE 95] dans le déploiement du système en question [DEB 02]. Dans le système technique, l'ensemble des éléments de connaissances et de savoirs technologiques sont ainsi reliés et mis en cohérence à un niveau fonctionnel [BAI 14].

En reprenant la typologie entre brique de connaissances et architecture de connaissances [HEN 90], identifier un système technologique nécessite de distinguer, d'une part, les briques de connaissances qui composent ce système. Elles correspondent aux unités de connaissances, plus ou moins 
importantes, qui sont associées au sein du système. D'autre part, les architectures de connaissances correspondent à la manière dont les briques de connaissances sont articulées entre elles et relèvent ainsi des liens qui existent entre les différentes briques.

Afin d'observer ces connaissances, deux logiques s'opposent. La première consiste à identifier les systèmes à partir de l'output, c'est-à-dire les artefacts technologiques associés au système et à retrouver quelles sont les connaissances qui le soutiennent. L'autre manière de procéder part des connaissances. Cela consiste à analyser les interacions entre les connaissances et ensuite à identifier les artefacts qui sont le fuit de ces interactions.

La première méthode ne semble pas appropriée à un travail sur la défense. En effet, pour des raisons de souveraineté, les innovations de défense sont souvent difficiles d'accès et leurs caractéristiques technologiques le sont encore plus. Les données sont par définition sensibles ; connaître dans le détail les caractéristiques d'un système d'arme particulier afin de le rattacher à un système technique plus large pose des problèmes évidents de sécurité si l'information est diffusée [BUE 11].

La méthodologie développée ici repose sur la seconde logique qui semble plus appropriée. Elle se fonde sur l'analyse des connaissances afin de délimiter des systèmes technologiques. Cela implique que la connaissance exhaustive des matériels militaires n'est pas nécessaire ; les données portant sur les connaissances, notamment grâce aux brevets, s'avèrent bien plus faciles à recueillir et à analyser pour un économiste. De plus comme il s'agit ici d'analyser le périmètre de l'innovation de défense afin de faciliter son intégration dans le paysage technologique global, les problèmes de souveraineté qui pourraient poser des questions sur la pertinence de l'utilisation de brevets s'éliminent. En effet pour ces technologies les Etats veillent, avec plus ou moins de succès, à ce que ces technologies n'échappent pas au périmètre qu'ils maitrisent et à ce que les industriels gardent le secret. Pour les autres technologies, la question de dépôt de brevets relève, comme dans toute industrie, de la stratégie d'entreprise. La démarche proposée ici consiste donc à étudier les interactions entre les connaissances afin de déterminer quelles sont celles qui font système et qui permettent de produire des innovations technologiques.

C'est à cette fin que le concept de SCT est proposé. Il se définit à l'image d'un système de connaissances scientifiques qui correspond à l'ensemble des connaissances étroitement liées les unes aux autres permettant l'élaboration et la compréhension d'une problématique scientifique. Ainsi, Cournot décrit par exemple les mathématiques comme un système de connaissances scientifiques « fondé sur des notions idéales qui se trouvent dans tous les esprits » [COU 47].

Dans cet esprit, un SCT correspond à l'ensemble des connaissances qui, étroitement liées entre elles, sont à l'origine de synergies dans la production technologique. Cet ensemble cohérent de connaissances (fondées sur des principes scientifiques ou non) associé à des compétences spécifiques permet de proposer des solutions techniques qui sont alors combinées entre elles au sein d'un ou plusieurs systèmes technologiques.

Afin de représenter les connaissances des entreprises $A \& D$, le concept de base de connaissances tel qu'il est défini par [NES 05, 06] ou [KRA 11] est utilisé. Une base de connaissances correspond au réseau des classes technologiques des brevets qu'une ou plusieurs organisation (s) dépose (nt). Dans le cas de cet article des entreprises du secteur A\&D. Ce réseau ou graphe est caractérisé par la matrice d'adjacence dont les caractéristiques seront étudiées par la suite.

\section{Données}

L'exploitation des données de brevets a d'ores et déjà démontré sa pertinence dans l'étude de la connaissance [TRA 02] [VER 04]. [LEY 14] soulignent que les brevets fournissent une source d'information riche sur l'activité d'innovation et son contexte en précisant notamment le nom des 
inventeurs et des exploitants, les sources de leurs connaissances ou encore les lieux où se déroule l'activité d'innovation. L'utilisation d'indicateurs structuraux permet d'analyser la production de la connaissance et sa diffusion. A l'instar de nombreux auteurs, cet article participe au développement nécessaire des méthodes qui permettent d'étudier ces données.

Les citations contenues dans les brevets permettent la construction de matrices de flux technologiques [VER 04]. Ces matrices sont alors utiliser pour étudier la production technologique. C'est afin de tirer le meilleur parti des données de brevets, tout en ayant conscience des limites qui y sont associées, que la methode proposée ici repose sur la constitution d'une base de connaissances en considérerant un ensemble de brevets issu des entreprises du secteur A\&D. En effet, la part formelle des connaissances s'avère relativement facile à observer puisque par définition il en existe une trace au travers des brevets par exemple ; la part tacite quant à elle l'est beaucoup moins.

La connaissance tacite n'est pas matérialisée en tant que telle. Alors, en observant l'organisation des connaissances formalisées entre elles dans diverses réalisations, il est possible de révéler la part informelle sous-jacente, car c'est en partie grâce à elle que les connaissances formalisées peuvent s'associer entre elles afin de produire une technologie. Dès lors, mieux comprendre la structuration de la connaissance constitue un enjeu, pour les entreprises elles-mêmes, mais aussi pour l'ensemble des acteurs qui cherchent à comprendre le processus de production et de diffusion de la connaissance (décideurs publics, économistes, régulateurs etc.). Cette articulation des connaissances formalisées peut s'observer à différentes échelles, au niveau d'un système technologique, d'une organisation, d'une industrie et plus spécifiquement dans le cas présent au niveau d'une base de connaissances.

Cet article s'appuie sur la base EC-JRC/OECD COR\&DIP qui répertorie les dépôts de brevets de 2000 groupes représentant en 2012 environ 90\% de la dépense en Recherche et Développement (R\&D) privée dans le monde. Nous avons décidé de ne retenir que les brevets déposés à l'office européen des brevets (EPO), à l'office américain des brevets (USPTO) ou dans l'un des offices des pays de l'Europe des 15 .

Comme la partie précédente de cet article a permis de le souligner, obtenir la liste des connaissances contenues dans un système technologique de défense pose plusieurs problèmes. L'un d'entre eux réside dans le caractère dual des entreprises A\&D. En effet, ces entreprises ne sont pas des pure playeur de la défense et la part de leur activité rattachée à ce domaine varie très fortement d'une entreprise à l'autre. Le choix qui a été fait est de concentrer l'analyse sur les entreprises qui au sein de la base COR\&DIP ont une activité dans le domaine de la défense et de considérer la contribution de ces entreprises à la base de connaissances défense en fonction de leur activité dans ce domaine ${ }^{1}$. Ces entreprises sont identifiées à l'aide des données du SIPRI ${ }^{2}$ (Top 100 compagnies data in a time series from 2002-15). Cette base de données répertorie chaque année le top 100 des entreprises ayant une activité dans le domaine de la défense. Entre 2010 et 2012, 65 entreprises appartiennent à la fois au top 100 SIPRI et à la base COR\&DIP. Ces entreprises comptent pour 368254 brevets déposés.

Ici, il est considéré que les flux technologiques réalisés au sein d'une entreprise, dont la part de l'activité défense est minime, ne peuvent être considérés à la même échelle que les flux observés dans une entreprise où la défense représente la totalité (ou presque) de son activité. C'est pourquoi, à la manière de Piscitello dans ses analyses sur la diversification des firmes, les résultats de l'analyse des bases de connaissances des firmes sont pondérés par la part que représente l'activité défense dans le chiffre d'affaires de l'entreprise [PIS 05]. Cela permet de donner une valeur plus importante aux liaisons technologiques réalisées dans une entreprise où l'activité de défense est importante. Cette démarche permet de mettre en avant le caractère défense des liaisons technologiques spécifiques à l'activité de défense au sein des bases de connaissances des entreprises.

Pour respecter ce principe, la valeur des coefficients de la matrice de flux technologiques relatifs à une entreprise est considérée comme une «couche». Chacune des couches représentant la base de 
connaissances d'une entreprise est pondérée par le pourcentage du chiffre d'affaires de chaque entreprise relative à l'activité défense. Pour cela, les données éditées par le SIPRI permettent de connaitre cette part de chiffre d'affaires chez les cent plus grands industriels de défense et ainsi de pondérer les résultats.

La matrice obtenue constitue alors une portion du système Input-Output global correspondant à la proportion de la défense au sein de ces flux technologiques. Elle représente les flux technologiques de défense et par conséquent la base de connaissances de défense au sein duquel sont identifiés les SCT.

Cette matrice est interprétée comme la matrice d'adjacence du graphe qui retrace les relations entre les différentes classes technologiques de la base de connaissances de défense. En reprenant la typologie entre brique de connaissances et architecture de connaissances [HEN 90], un tel graphe distingue les briques de connaissances qui correspondent aux classes technologiques et les architectures de connaissances qui sont représentées par les liens entre les classes technologiques.

\section{Méthode}

Dans cette partie, nous présentons les résultats empiriques de notre étude. Premièrement, est développé une analyse descriptive des données de famille de brevets du domaine $\mathrm{A} \& \mathrm{D}$, en particulier un compte rendu de l'utilisation de connaissances scientifiques dans ces familles. Dans un second temps, nous approfondissons cette étude descriptive en introduisant les corrélations entre la qualité des brevets et la NPL.

La méthode s'ancre dans le cadre de la Théorie de la Dominance Economique (TDE) [PER 48] et mobilise les outils de la Théorie des Graphes d'Influence (TGI) qui en découlent [LAN 74]. Afin de mesurer les synergies dans la production de connaissances technologiques nous structurons les données sous la forme d'un système Input-Output (IO) en utilisant les citations entre les brevets [JAF 02]. Ce système se matérialise sous la forme d'un graphe et d'une matrice carrée reprenant les 641 classes technologiques de la Classification Internationale des Brevets (CIB). Chaque entreprise considérée au prorata de son chiffre d'affaires dans le domaine de la défense permet d'isoler les flux de connaissances liés à ce domaine. C'est à l'aide de ces données que la base de connaissances défense est constituée afin de mesurer les interdépendances entre les classes technologiques (les pôles du graphe) pour mettre en évidence des synergies et ainsi définir des SCT.

Comme le rappellent [LEB 15], la Théorie de la Dominance Economique (TDE) repose sur deux ancrages, l'un théorique et l'autre méthodologique. Théoriquement, la TDE se situe dans la filiation des travaux menés par F. Perroux sur les relations de pouvoir (de dominance) en économie. Ces travaux proposent une conception du monde économique, comme un ensemble de rapports de domination qui peuvent être patents ou dissimulés [PER 48]. Méthodologiquement, elle se rattache à la théorie mathématique des graphes qui constitue l'outil privilégié des études sociométriques, autrement appelées, Analyse des Réseaux Sociaux (ARS). L.C. Freeman souligne que "l'école de la Sorbonne », établit une première synthèse de ces écrits "montrant explicitement qu'un vaste champ de problèmes sociaux pouvait être compris comme des cas particuliers d'un modèle structural général » [FRE 04].

Jusqu'à présent la TDE s'est principalement appliquée à l'analyse des relations interindustrielles. D'abord, dans un cadre national, afin d'étudier les relations de dépendance et d'interdépendance entre les branches productives telles que la comptabilité nationale les représente [LAN 74] et, ensuite, dans une perspective internationale, afin d'étudier les relations de domination dans le cadre des échanges internationaux [LEB 15]. En se fondant sur la TDE, cet article propose d'analyser la production de connaissances afin de comprendre les relations de dépendance et d'interdépendance qui la structurent. Pour cela, la base de connaissance défense est définie à l'aide des données de brevets. 
L'exploitation des données de citations de brevets permettent de construire des matrices de flux technologiques (cf. [VER 04] pour plus de précisions sur la construction des matrices de flux). Ces citations de brevets permettent d'analyser les relations inter technologiques durant le processus d'innovation [AUT 14]. L'intérêt est de pouvoir tracer un lien orienté et pondéré entre des classes technologiques «citantes» (celles auxquelles sont rattachés les brevets «citants») et les classes technologiques citées (celles auxquelles sont rattachés les brevets cités). A ce stade nous précisons trois pondérations qui sont opérées lors de la construction des matrices.

Premièrement, la pondération interne au brevet : les liens technologiques entre les classes citées et «citantes » sont comptabilisées comme une fraction du nombre de combinaisons possibles compte tenu du nombre de classes technologiques référencées dans chaque brevet.

Deuxièmement, la pondération liée aux entreprises détentrices du brevet. Si le brevet en question n'est détenu que par une seule entreprise alors il n'est pas concerné par cette pondération. En revanche si plusieurs entreprises se partagent la propriété d'un brevet, alors la valeur des flux technologiques qui lui sont associés est répartie équitablement entre les différentes entreprises (fractionnal counting).

Troisièmement, la pondération liée aux familles de brevets. Ce travail portant sur plusieurs offices de protection de la propriété intellectuelle, nous n'observons pas simplement les brevets, mais plus exactement les familles de brevets. Ces familles permettent de regrouper plusieurs brevets déposés dans différents offices mais faisant référence à la même Invention. En effet, une même invention peut faire l'objet d'un brevet aux Etats-Unis, d'un autre en Europe, d'un autre spécifiquement pour l'office Français, etc. Chaque brevet répertorie alors une liste de Classes Technologiques (CT) « citantes » et de CT « citées ». Or, chaque office est plus ou moins indépendant et procède selon ses propres méthodes pour identifier les $\mathrm{CT}$ «citantes » ainsi que les brevets cités et donc les $\mathrm{CT}$ « citées » pour chaque brevet [MER 09] ce qui conduit, d'un brevet à l'autre, à des écarts dans la liste des classes technologiques, alors même qu'ils appartiennent à la même famille. Il convient donc, lors de l'agrégation ces brevets, de ne point surestimer ou sous-estimer la valeur des liaisons technologiques. Pour cela nous ne prenons en compte, comme c'est communément le cas, que les liaisons uniques dans chaque brevet (exemple : un brevet américain citant un brevet allemand et un brevet Français, de la même famille que le brevet américain, ayant les mêmes CT « citantes » et qui cite le même brevet allemand, regroupent des citations identiques qui ne doivent alors être comptées qu'une seule fois). En effet, nous considérons que les doublons font référence aux mêmes liens technologiques.

Le premier bénéfice de l'analyse des matrices de flux technologiques à l'aide de la TDE est l'identification des synergies entre les connaissances et par conséquent le repérage de SCT au sein d'une base de connaissances.

Le principe de la Théorie des Graphes d'Influence TGI qui est exploité ici consiste à dire que lorsque l'on considère un système Input Output dont on calcule la matrice de Leontief ou la matrice de Gosh, la valeur des interdépendances au sein de la structure est égale à $\boldsymbol{I}_{G}=\mathbf{1}-\boldsymbol{D}$ avec $\boldsymbol{I}_{\boldsymbol{G}}$ l'interdépendance générale et $\mathrm{D}$ le déterminant de la structure. Afin de respecter les théorèmes de la TGI les liens avec l'extérieur et l'auto consommation des pôles sont considérés comme nuls [LAN 15]. Ici l'objectif étant d'analyser l'interdépendance entre les pôles ces précautions n'ont pas d'incidence sur les résultats tout en simplifiant les calculs.

Afin de mesurer les interdépendances entre les pôles d'une structure à l'aide de la TGI, une méthode a été développée par le passé [LEB 09], elle consiste en une analyse de robustesse dont le principe est de procéder par itération. L'idée est, en partant de la structure complète, de supprimer à chaque tour l'arc, qui une fois déduit de la structure, permet de maximiser l'interdépendance globale de la structure restante. Ainsi, le premier arc retiré est celui qui contribue le moins à l'interdépendance générale, alors que le dernier retiré est celui qui y contribue le plus. En classant ces arcs du dernier au premier, il est 
possible de reconstruire une structure mettant en avant les relations les plus fortes entre les pôles ce qui fait émerger des clusters.

Néanmoins, cette procédure est à la fois longue, fastidieuse et consomme beaucoup de ressources informatiques. En effet, à chaque itération, il faut tester la suppression de tous les arcs afin de définir lequel doit être supprimé. L'enjeu méthodologique est de proposer ici une procédure alternative permettant de faire émerger des interdépendances entre les pôles pour identifier des SCT.

Pour cela, au sein d'une structure, ou l'autarcie est nulle, une partition P est définie telle que : $\boldsymbol{p}_{\mathbf{1}}$ est une partie principale constituée de 2 pôles et $p_{2}$ une partie complémentaire constituée du reste de la structure à n- 2 pôles. Alors, pour chacune des partitions $\mathrm{P}$ possibles, on peut montrer que la somme des interdépendances intra et inter-partie est supérieure ou égale à l'interdépendance générale de la structure. En effet, avec l'interdépendance générale $\boldsymbol{I}_{G}=\mathbf{1}-\boldsymbol{D}$ et d'après le théorème de la partition [LAN 13], la somme des interdépendances intra et inter-partie est égale à :

$$
\begin{aligned}
& \sum_{i}^{2}\left(1-D_{p i}\right)+D_{p 1} D_{p 2}-D \\
& =1-D_{p 1}-D_{p 2}+D_{p 1} D_{p 2}+(1-D) \\
& =\prod_{i}^{2}\left(1-D_{p i}\right)+I_{G}
\end{aligned}
$$

Or, étant donné que $\left(\mathbf{1}-\boldsymbol{D}_{p i}\right) \geq \mathbf{0}$, alors :

$\prod_{i}^{2}\left(1-D_{p i}\right) \geq 0$

Donc, avec $\boldsymbol{I}_{G} \geq 0$ :

$\prod_{i}^{2}\left(1-D_{p i}\right)+I_{G} \geq 0$

Le surplus par rapport à $\boldsymbol{I}_{G}$ mesure une synergie $\boldsymbol{S}_{P}$ associée à une certaine partition $\mathrm{P}$ et correspond au poids des interdépendances internalisées dans les deux parties.

$$
S_{P}=\prod_{i}^{2}\left(1-D_{p i}\right)
$$

Considérons deux cas :

Si on pose le cas où, les interdépendances au sein de la partie complémentaire sont nulles. Alors, la somme des interdépendances dans la partie principale $\left(\mathbf{1}-\boldsymbol{D}_{p \mathbf{1}}\right)$ et les interdépendances entre les parties $\left(\boldsymbol{D}_{\boldsymbol{p} 1}-\boldsymbol{D}\right)$ est :

$$
\left(1-D_{p 1}\right)+\left(D_{p 1}-D\right)=I_{G}
$$

De même, s'il n'y a aucune interdépendance au sein de la partie complémentaire, alors, la somme des interdépendances dans la partie complémentaire $\left(\mathbf{1}-\boldsymbol{D}_{p 2}\right)$ et les interdépendances entre les parties $\left(D_{p 2}-D\right)$ est :

$$
\left(1-D_{p 2}\right)+\left(D_{p 2}-D\right)=I_{G}
$$

Dans ces deux cas, il n'y a aucune synergie liée à la partition envisagée, et donc aucun surplus : 


\section{$S_{P}=0$}

Une synergie $S_{P}>\mathbf{0}$ correspond à une situation où il existe simultanément, pour une partition $P$, des interdépendances à l'intérieur des deux parties. Trouver les synergies $\boldsymbol{S}_{\boldsymbol{P}}$ les plus fortes revient à trouver les combinaisons de $\mathbf{1}-\boldsymbol{D}_{p \mathbf{1}}$ et $\mathbf{1}-\boldsymbol{D}_{p \mathbf{2}}$ les plus fortes, i.e. les interdépendances dans la partie principale au regard de la valeurs des interdépendances dans la partie complémentaire.

L'intérêt de la méthode est de calculer, pour chacune des combinaisons de deux pôles, les synergies qu'elles engendrent en tenant compte, à la fois, des interdépendances entres ces pôles et des interdépendances dans le reste de la structure. Ainsi, toute chose égale par ailleurs, une combinaison de deux pôles qui nuit trop fortement au potentiel synergétique du reste de la structure (i.e qui diminue fortement le score d'interdépendance de la partie complémentaire) obtiendra un score de synergie moins élevé qu'une autre combinaison qui préserve plus de circularité dans le reste de la structure. Cela permet d'octroyer les scores de synergies les plus fortes aux combinaisons de deux pôles les plus interdépendants qui ne cassent pas de circularité dans la partie complémentaire. Cela définit en plein de fortes synergies entre les deux pôles et en creux une préservation du potentiel synergétique dans le reste de la structure. C'est ce score de synergie liée à une partition particulière qui permet de calculer un score de synergie deux à deux entre les pôles d'une structure.

Pour appliquer cette méthode à la définition des SCT, il suffit de l'appliquer à la matrice des coefficients technologiques de la base de connaissances défense. Puis de calculer pour toutes les combinaisons de classes technologiques les scores de synergie tels qu'ils viennent d'être définis.

\section{Résultats}

Afin de ne considérer que les technologies faisant partie du cœur de notre structure et ainsi d'éviter de prendre en compte, dans une trop grande mesure, les synergies associées au General Purpose technologies par exemple [BRE 95], nous décidons d'un seuil de coupe.

Pour cela, les scores d'interdépendance obtenus sont normés entre « 0 » et « $1 »$; le score de « 0 » représente la plus forte interdépendance entre deux pôles au sein de la structure et le score de «1» représente l'absence d'interdépendance. Sont alors obtenus des scores de synergie relative entre deux pôles. Donc, on ne retient que les interdépendances dont les scores sont inférieurs à un certain pourcentage du score maximum (l'interdépendance la plus faible). Par exemple, en choisissant un seuil de coupe à $35 \%$ ne sont sélectionnées que les synergies dont le score est inférieur à $35 \%$ du score le plus élevé.

Ces scores de synergie relative sont classés au sein d'une matrice symétrique. Elle s'interprète comme une matrice de distance dont les éléments de la diagonale sont égaux à 0 . Cette matrice se représente graphiquement à l'aide d'un dendrogramme dont la figure 1 présente une fraction. C'est en étudiant ce dendrogramme qui représente les synergies entre les classes technologiques de la base de connaissances défense que les SCT sont identifiés. 


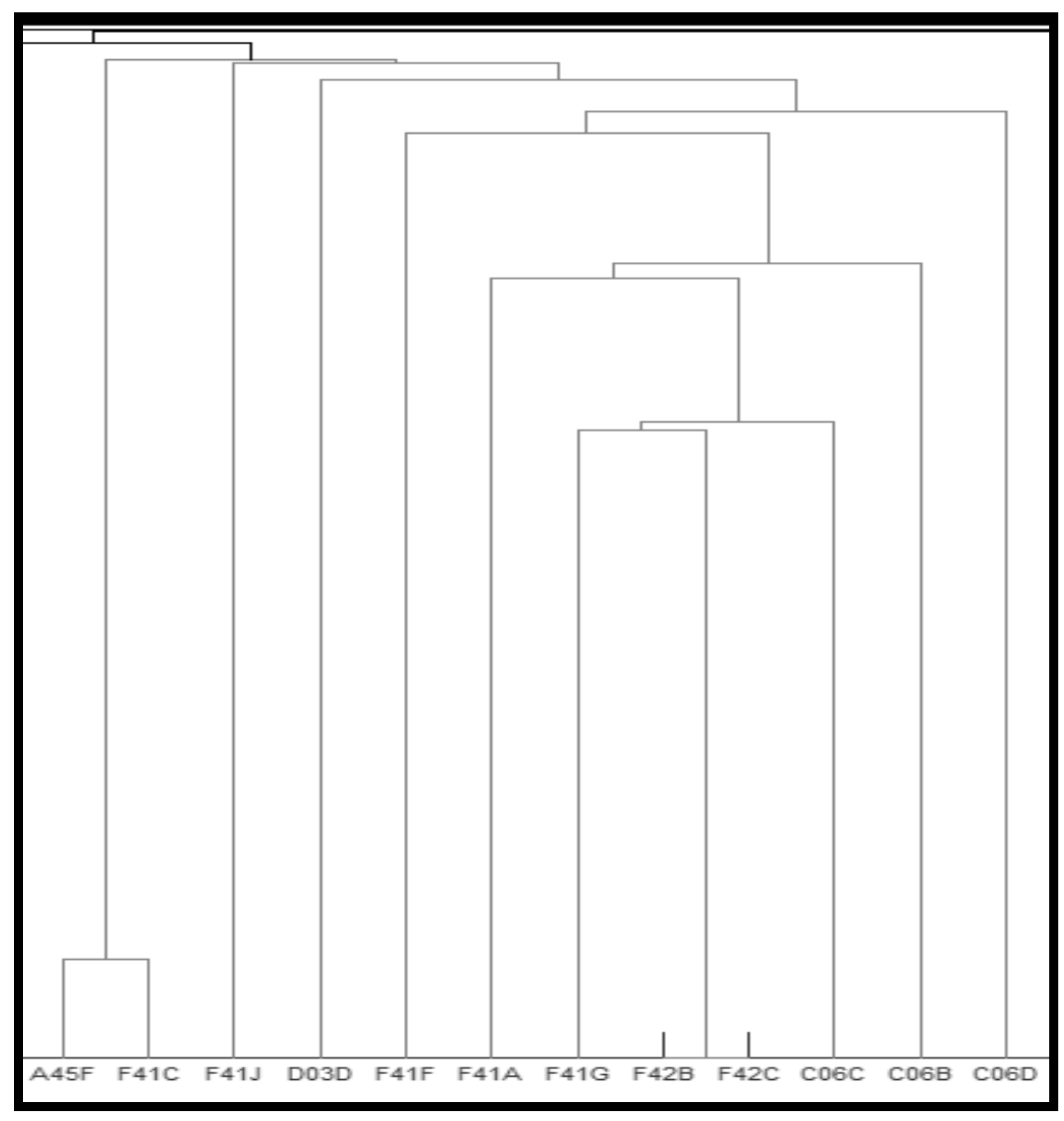

Figure 1. Portion de dendrogramme représentant un SCT

Afin d'obtenir les résultats les plus pertinents possibles, une procédure par itération successive a été choisie, chose rendue possible par les économies en ressources informatiques réalisées. Chaque itération correspond à un niveau de coupe. Plus le seuil de coupe est bas, plus le nombre de technologies incluses dans l'analyse est grand. Il est de plus en plus bas à mesure que plus de classes technologiques souhaitent être incluses dans l'analyse. Ici, le niveau de coupe le plus fin choisi est de $35 \%$ du score maximum puis est diminué par tranche de 5\% et finit, au niveau le plus fin, à un score de $1 \%$. D'une part, ceci permet de porter, en priorité, l'attention sur les synergies les plus fortes au sein de la base de connaissances technologiques de défense (douze technologies au seuil de 35\%). D'autre part, cela permet de visualiser les interdépendances qui se greffent aux premières à mesure que le nombre de technologies étudiées augmente. In fine, ces deux éléments permettent de choisir le niveau de coupe le plus pertinent pour chaque SCT. Dans une telle démarche, les SCT dont l'ensemble des synergies apparaissent aux seuils de coupe les plus élevés sont les plus synergétiques dans la base de connaissances défense et donc ceux qui en sont le plus caractéristique.

L'application de la méthode a permis d'identifier 26 SCT sur la base des synergies observées au sein de la base de connaissances défense entre 2010 et 2012. Ils recouvrent des domaines technologiques diversifiés qui dépassent les classes technologiques appartement au sous domaine 28 consacré aux technologies « spatial et armement» identifié pas l'Observatoire des Sciences et Techniques (OST).

Ces 26 SCT sont répertoriés dans le tableau 1, ils y sont classés en fonction de la force de leurs synergies, c'est-à-dire en fonction du niveau de coupe auxquels ils apparaissent. Le nom de chacun des $\mathrm{SCT}$ est donné à titre indicatif, il fait référence au domaine d'application le plus évident, mais tous les SCT peuvent par définition trouver des applications dans différents systèmes technologiques. 


\begin{tabular}{|c|c|c|c|}
\hline SCT & Technologies & Numéro classe technologiques & $\begin{array}{l}\text { Nombre de } \\
\text { technologies }\end{array}$ \\
\hline 1 & Missiles & F41F F41A F41G F42B F42C C06B C06C C06D & 8 \\
\hline 2 & Armement* & $\begin{array}{l}\text { A45F F41C F41J D03D F41F F41A F41G F42B F42C C06B } \\
\text { C06C C06D }\end{array}$ & 12 \\
\hline 3 & Réacteurs et turbines & $\begin{array}{l}\text { F03B F16J F23C F02K F04D F01D F02C F23R F23D F23N } \\
\text { F24H F02G }\end{array}$ & 12 \\
\hline 4 & Véhicule de défense & F41H B60D B62D B60G F16F & 5 \\
\hline 5 & Communication & G06Q G06F H04L H04B H04W H04J & 6 \\
\hline 6 & Guidage & H01P H01Q G01S G01C G08G G05D & 6 \\
\hline 7 & Aéronef & B64C B64D & 2 \\
\hline 8 & Guidage à distance* & $\begin{array}{l}\text { G06Q G06F H04L H04B H04W H04J H03H H01P H01Q } \\
\text { G01S G01C G08G G05D }\end{array}$ & 13 \\
\hline 9 & Drone* & $\begin{array}{c}\text { H03G H03F H04M H03D H04K G05B G06N G06Q G06F } \\
\text { H04L H04B H04W H04J H03H H01P H01Q G01S G01C } \\
\text { G08G G05D B64C B64D }\end{array}$ & 25 \\
\hline 10 & Drone de défense* & $\begin{array}{l}\text { F41H B60D B62D B60G F16F H03G H03F H04M H03D } \\
\text { H04K G05B G06N G06Q G06F H04L H04B H04W H04J } \\
\text { H03H H01P H01Q G01S G01C G08G G05D B64C B64D }\end{array}$ & 30 \\
\hline 11 & Pétrochimie & B01D B01J C07C C10G C10L C07B C01B & 8 \\
\hline 12 & Pneumatiques & B29B B32B B29C B29D B60C C08K C08L C08F D02G & 10 \\
\hline 13 & $\begin{array}{l}\text { Train roulant du } \\
\text { véhicule* }\end{array}$ & $\begin{array}{l}\text { B29K B28B B29B B32B B29C B29D B60C C08K C08L } \\
\text { C08F D02G D07B C08C B29L C08J C08G }\end{array}$ & 16 \\
\hline 14 & Systèmes électriques & H02J H02M G05F H02P & 4 \\
\hline 15 & Moteurs électriques* & H01M H02J H02M G05F H02P H02K F03D H02H B60L & 9 \\
\hline 16 & Véhicule en général & B60W B60K F16H F16D B60T & 5 \\
\hline 17 & Véhicule électrique* & $\begin{array}{l}\text { H01M H02J H02M G05F H02P H02K F03D H02H B60L } \\
\text { B60W B60K F16H F16D B60T }\end{array}$ & 14 \\
\hline 18 & $\begin{array}{l}\text { Optique, traitement } \\
\text { des données images }\end{array}$ & G01N G01J H01S G02B G02F H04N G06K G06T & 8 \\
\hline 19 & Electronique & H01L H05K H01R H02G & 4 \\
\hline 20 & Capteurs* & $\begin{array}{c}\text { G01N G01J H01S G02B G02F H04N G06K G06T G03B } \\
\text { H01L H05K H01R H02G }\end{array}$ & 12 \\
\hline 21 & Biochimie & C12M C12P C12N C12Q C07H C40B & 6 \\
\hline 22 & Signaux lumineux & B60Q F21S F21V F21L F21Y & 5 \\
\hline 23 & $\begin{array}{l}\text { Aménagement du } \\
\text { véhicule }\end{array}$ & A47C B60N B60R E06C & 4 \\
\hline 24 & Travail des métaux & B21B C21D C22C C22F B22F & 5 \\
\hline 25 & Moteur à combustion & F16N F01M F01L F02D F02B F02M F16K F01N & 8 \\
\hline 26 & Travail des métaux & B23C B24D B24B B23F & 4 \\
\hline
\end{tabular}


Les SCT sont de deux catégories, la première est constituée des SCT simples, c'est-à-dire indépendants de tout autre SCT, la seconde regroupe des SCT complexes, c'est-à-dire qu'ils sont composés de plusieurs SCT regroupés ensemble (toujours en fonction de la force des synergies). Ces deux niveaux de SCT permettent d'appréhender les synergies de connaissances à différents niveaux et permet ainsi de mettre en évidence que des regroupements technologiques identifiés à différentes échelles peuvent être reliés à différents systèmes technologiques. Les SCT complexes sont notés d'une étoile dans le tableau ci-dessus.

Ainsi la mise en évidence de SCT faisant référence aux systèmes de communications (SCT5) et aux systèmes de guidages (SCT6) montre que les industriels de défense ont un rôle à jouer dans le développement de ces technologies mais le fait qu'elles soient principalement synergétiques avec le SCT lié aux aéronefs (SCT7) montre que cette implication a principalement à voir avec le développement du Drone. Les synergies entres ces 3 SCT et le SCT4, seul SCT dont les classes technologiques font explicitement référence au domaine d'application défense (blindage; tourelles cuirassées; véhicules blindés ou armés; moyens d'attaque ou de défense, p.ex. camouflage, en général) montre que dans ces domaines d'innovation la spécificité défense ne constitue pas le cœur du système mais au contraire une de ses caractéristiques périphériques. Cela concorde avec le caractère dual que l'on pouvait anticiper pour ces technologies.

Les SCT sont composés en moyenne de 9,2 technologies avec, un peu plus de 6,1 technologies pour les SCT simples et 16,3 technologies pour les SCT complexes. Comme attendu les SCT identifiés au plus haut niveau de synergie sont ceux dont la spécificité militaire est la plus forte (missiles et armement en général). Il n'est pourtant pas étonnant de trouver bon nombre de SCT dans des domaines de connaissances plus génériques tel que l'électronique, les systèmes de propulsion ou encore les véhicules. Les technologies fortement attachées au secteur aéronautique sont particulièrement représentées avec les SCT 3, 7, 9 et 10. Ceci est logique en raison de la sélection des firmes en fonction de leur appartenance au secteur A\&D. Néanmoins, ne pas se cantonner aux entreprises de ce secteur permet de mettre en avant une plus grande diversité technologique au sein de la base de connaissances défense.

La mise en évidence des SCT caractéristiques de l'innovation de défense permet dans un premier temps de mieux comprendre les synergies qui structurent son innovation mais cela permet aussi d'envisager la manière dont ces ensembles synergétiques peuvent interagir avec le reste du paysage technologique. Depuis les années 1980, le contexte dans lequel l'innovation de défense évolue est de plus en plus dual [MEN 17], marqué par la complexité des matériels [HOB 05], par la hausse des coûts [SER 08] et par la fin des logiques d'arsenal et la privatisation des entreprises [BLA 00]. Ce nouvel environnement pousse les entreprises, dans le respect des contraintes de la souveraineté nationale, à plus d'ouverture technologique en raison de la dualité et à plus d'ouverture territoriale, en raison de l'internationalisation des firmes. Dans ce contexte, il est devenu plus difficile d'imaginer que la Recherche et Développement (R\&D) militaire se développe en ignorant son environnement. C'est l'intégration à cet environnement qui offre de nouvelles opportunités d'innovation. Cela est lié au fait que la production de connaissances est un processus cumulatif et un processus fondé sur une structure co-relationnelle [KRA 11].

Un processus cumulatif. Autrement dit la connaissances que l'on est capable de produire dépend des connaissances que l'on a assimilées par le passé.

Un processus reposant sur une structure co-relationnelle. La maitrise d'une connaissance supplémentaire permet de multiplier les combinaisons de connaissances et donc d'augmenter les potentiels d'innovation d'autant [FLE 01].

Afin de pouvoir s'intégrer avec son environnement, l'innovation de défense doit partager avec ce dernier un certain nombre de connaissances qui constituent le socle de cette intégration. La question de 
la diffusion de la connaissance est primordiale dans un processus d'intégration technologique. Or, la diffusion des connaissances dépend de l'adéquation des connaissances que l'organisation maitrise et celles qu'elle désire. C'est ce qui détermine les capacités d'absorption des firmes [COH 90]. C'est pour cela qu'il est important de connaître les caractéristiques de l'innovation de défense pour savoir, d'une part, quelles sont ses capacités d'absorption i.e. les connaissances qu'elle est capable d'assimiler et, d'autre part, si les capacités d'absorption du reste du paysage technologique permettent d'assimiler les connaissance produites dans le cadre de l'innovation de défense. Mieux connaître la nature des connaissances produites au sein de la base de connaissances de défense permet donc de mieux comprendre son potentiel d'intégration.

Dans cette optique, la typologie proposée par [HEN 90] permet de différencier la brique de connaissances (component knowledge) qui correspond à l'unité de connaissance qui peut être plus ou moins importante, de l'architecture de connaissances (architectural knowledge) qui révèle les relations qui existent entre elles. Or ces deux types de connaissances peuvent se retrouver à d'autres niveaux. D'abord, il est possible d'analyser la production des connaissances au sein d'une organisation à l'aide de la notion de base de connaissances déjà évoquée. Elle permet de mettre en évidence les connaissances individuelles qu'une organisation mobilise mais aussi la manière dont elle les organise. Ensuite, à la manière de [ULR 95], il est possible d'étudier les briques de connaissances et l'architecture de connaissances au niveau des produits. Cette architecture se comprend dans ce cas comme le schéma qui lie ces composants entre eux. Le SCT constitue une manière d'identifier les briques et l'architecture de connaissances en s'émancipant de l'indentification du produit.

Chaque SCT correspond à une liste de briques de connaissances liées entre elles par une architecture mise en évidence par des synergies technologiques (citations). Il constitue ainsi un instrument permettant de repérer les proximités de connaissances qui peuvent exister entre un SCT et n'importe quelle base de connaissances au niveau d'une organisation, d'un secteur industriel ou même d'un territoire. Ces proximités constituent le socle à partir duquel l'innovation de défense peut développer son intégration tant technologique que géographique.

\section{Conclusion}

La TDE et plus particulièrement la TGI offre un ensemble d'outils pour analyser une structure d'échanges. Appliqués à l'analyse des flux de connaissances matérialisés à partir des citations de brevets, ces outils permettent de mettre en évidence les relations de dépendance, d'interdépendance, et de dominance dans le processus de production de la connaissance.

Cette première application de ces outils à la base de connaissances défense a permis de révéler des synergies associées à l'activité de défense au sein des entreprises. Les 26 SCT que cela a permis d'identifier constitueront le matériau empirique de base à partir duquel d'autres études pourront être menées afin de déterminer les territoires, les secteurs industriels, les technologies ou encore les entreprises au sein desquels l'innovation de défense est susceptible de pouvoir s'intégrer et vice-versa.

\section{Bibliographie}

[ALI 92] Alic, J. A., Beyond Spinoff: Military and Commercial Technologies in a Changing World, Harvard Business Press, 1992.

[AUT 14] Autant-Bernard C., Cowan R., Massard N., « Editors' introduction to spatial knowledge networks: structure, driving forces and innovative performances », The Annals of Regional Science, $\mathrm{n}^{\circ} 53$ (2), p. 315-323, 2014.

[BAI 13] Bainee J., Conditions d'émergence et de diffusion de l'automobile électrique : une analyse en termes de "biensystème territorialisé", Thèse de doctorat, Université Paris 1 - Panthéon-Sorbonne, 2013.

[BIJ 10] Bijker W. E., « How is technology made? That is the question! », Cambridge Journal of Economics, vol. 34, ${ }^{\circ}$ 1, p. 63-76, 2010. 
[BLA 00] Blanc G., « Dépenses militaires, restructuration de l'industrie d'armement et privatisation de la défense : analyse comparée France-Etats-Unis, 1994-1999: analyse comparée France-Etats-Unis, 1994-1999 », Arès, $n^{\circ}$ 46, 2000 .

[BRE 95] Bresnahan T. F., Trajtenberg M, « General purpose technologies 'Engines of growth'?, », Journal of Econometrics, vol. 65, n 1, p. 83-108, 1995.

[BUE 01] Buesa M, "Controlling the international exchanges of armaments and dual - use technologies: The case of Spain », Defence and Peace Economics, vol. 12, n 5, p. 439-464, 2001.

[CAR 91] Carlsson B., Stankiewicz R., " On the nature, function and composition of technological systems », J Evol Econ, vol. 1, n 2, p. 93-118, 1991.

[COH 89] Cohen W. M., Levinthal D. A., «Innovation and learning: the two faces of R \& D », The economic journal, p. 569-596, 1989.

[COU 47] Cournot A. A., De l'origine et des limites de la correspondance entre l'algèbre et la géométrie, L. Hachette, Paris, 1847.

[DEB 02] De Bandt J., "L'émergence du nouveau système technique ou socio-technique », Revue d'économie industrielle, vol. 100, $\mathrm{n}^{\circ} 1$, pp. 9-38, 2002.

[FLE 01] Fleming L., Sorenson O., « Technology as a complex adaptive system: evidence from patent data », Research Policy, vol. 30, n 7, p. 1019-1039, 2001.

[FRE 97] Freeman C., Soete L., The economics of industrial innovation, Psychology Press, Hove, 1997.

[GIL 78] Gille B., Histoire des Techniques : techniques et civilisation, technique et sciences, Encyclopédie de la Pléiade, Paris, 1978.

[GUM 88] Gummett P., Reppy J. (dir), The Relations between Defence and Civil Technologies, Dordrecht: Springer Netherlands, 1988.

[HEN 90] Henderson R. M., Clark K. B., « Architectural Innovation: The Reconfiguration of Existing Product Technologies and the Failure of Established Firms », Administrative Science Quarterly, vol. 35, n 1, p. 9, 1990.

[HOB 05] Hobday M., Davies A., Prencipe A, «Systems integration: a core capability of the modern corporation », ICC, vol. 14, n . 6, p. 1109-1143, 2005.

[JAF 02] Jaffe A.B., Trajtenberg M., Patents, Citations, and Innovations: A Window on the Knowledge Economy, MIT Press, Cambridge, 2002.

[KRA 11] Krafft J., Quatraro F., Saviottti P-P., « The Knowledge Base Evolution in Biotechnology: A Social Network Analysis », Taylor \& Francis, n 5, p.445-475, 2011.

[LAN 74] Lantner R., Théorie de la dominance économique, Dunod, Paris, 1974.

[LAN 15] Lantner R., Lebert D., « Dominance et amplification des influences dans les structures linéaires », Économie appliquée - ISMEA, vol. 68, n 3 p.143-165, 2015.

[LEB 09] Lebert D, Younsi H. El., Lequeux F., Gallo J., Zyla E., « Les échanges industriels entre les pays du bassin méditerranéen : application d'un nouvel algorithme de clustering sur données de flux », dans H. Ben Hammouda, N. Oulmane, R. Sandretto (dir), Emergence en Méditerranée : attractivité, investissements internationaux et délocalisations, L'Harmattan, Paris, 2009.

[LEB 15] Lebert D., Younsi H. El., « Théorie de la dominance économique : indicateurs structuraux sur les relations interafricaines, » Économie appliquée — ISMEA, vol. 68, n³ 3, pp. 167-186, 2015.

[LEY 14] Leydesdorff L., Alkemade F., Heimeriks G., Hoekstra R., « Patents as instruments for exploring innovation dynamics: geographic and technological perspectives on "photovoltaic cells" », Scientometrics. 2014.

[MER 09] Merindol V., Fortune E., « Analyse des informations disponibles dans la base Patstat », OST - Observatoire des sciences et des techniques, 2009.

[MEU 17] Meunier F-X., L'innovation Technologique duale : une analyse en termes d'influence et de cohérence, Thèse de doctorat, Université Paris1 Panthéon Sorbonne, 2017

[NES 05] Nesta L., Saviotti P. P., « Coherence of the Knowledge Base and the Firm's Innovative Performance: Evidence from the U.s. Pharmaceutical Industry », The Journal of Industrial Economics, vol. 53, n 1, pp. 123-142, 2005.

[PER 48] Perroux F., « Esquisse d'une théorie de l'économie dominante », Économie appliquée- ISMEA, vol. 1, n 2 $3,1948$. 
[PIS 05] Piscitello L., « Corporate Diversification, Coherence and Firm Innovative Performance, » Revue d'économie industrielle Vol. 110. p. 127-148, 2005.

[SER 08] Serfati C., "Le rôle de l'innovation de Défense dans le système national d'innovation de la France », Innovations, vol. 28, $\mathrm{n}^{\circ}$ 2, p. 61, 2008.

[ULR 95] Ulrich K., « The role of product architecture in the manufacturing firm », Research policy, vol. 24, $\mathrm{n}^{\circ} 3, \mathrm{p}$. 419-440, 1995.

[VER 04] Verspagen B., «Structural Change and Technology: A Long View », Revue économique, vol. 55, $\mathrm{n}^{\circ}$ 6, p. 1099, 2004.

\footnotetext{
${ }^{1}$ Le poids de l'activité défense varie entre $1 \%$ et $100 \%$ du chiffre d'affaires de ces entreprises.

${ }^{2}$ Stockholm International Peace Research Institute (SIPRI).
} 